J. Lake Sci. (湖泊科学), 2016, 28(2): 328-333

DOI 10. $18307 / 2016.0212$

(c) 2016 by Journal of Lake Sciences

\title{
广州市湖泊水体中防腐剂浓度及其潜在毒性风险”
}

\author{
刘祖发 ${ }^{1,2}$, 关 帅 ${ }^{1,2}$, 林颖妍 ${ }^{1,2}$, 邓 哲 ${ }^{1,2}$, 丁 波 ${ }^{1,2}$,查悉妮 ${ }^{1,2}$ \\ (1: 中山大学水资源与环境研究中心,广州 510275 ) \\ (2:华南地区水循环与水安全广东省普通高校重点实验室,广州 510275)
}

\begin{abstract}
摘 要: 为研究广州市湖泊水体中的防腐剂及其毒性, 在广州市选择 15 个湖泊采集水样, 采用液液萃取-气质联用法对 湖泊水体中的对羟基苯甲酸甲酯、对羟基苯甲酸乙酯等 5 种防腐剂进行浓度检测和毒性分析. 结果表明:广州市区内 15 个湖泊水体中均检出防腐剂, 但其浓度略低于国外天然水体中的浓度, 溶解相的防腐剂平均浓度为 $5.06 \mathrm{ng} / \mathrm{L}$, 颗粒相的 平均浓度为 $0.78 \mathrm{ng} / \mathrm{L}$; 广州市区的湖泊水体中溶解相浓度最高的防腐剂为对羟基苯甲酸丁酯, 占总量的 $30.45 \%$, 对羟基 苯甲酸苯甲酯所占比例最小, 占 $6.71 \%$; 以防腐剂对发光细菌光强的抑制强弱来表征其毒性的大小, 对羟基苯甲酸苯甲 酯的毒性最强,对广州市湖泊水体环境的影响最大.
\end{abstract}

关键词: 湖泊;防腐剂;广州;气质联用; 毒性

\section{Research of parabens and its potential toxicity risk in lakes of Guangzhou}

\author{
LIU Zufa ${ }^{1,2}$, GUAN Shuai ${ }^{1,2}$, LIN Yingyan ${ }^{1,2}$, DENG Zhe ${ }^{1,2}$, DING Bo ${ }^{1,2}$ \& ZHA Xini ${ }^{1,2}$ \\ (1: Center for Water Resources and Environment, Sun Yat-sen University, Guangzhou 510275, P.R.China) \\ (2: Key Laboratory of Water Cycle and Water Security in Southern China of Guangdong Higher Education Institutes, Guang- \\ zhou 510275, P.R.China)
}

Abstract:In this study, the method of GC-MS with liquid-liquid extraction was adopted to analyze the parabens in 15 lakes of Guangzhou. The results showed that: parabens were detected from all the sampling sites while the content was not high contrasted with foreign waters. The average content of parabens in aquatic matter is $5.06 \mathrm{ng} / \mathrm{L}$, and that in suspended particulate matter is $0.78 \mathrm{ng} / \mathrm{L}$. Concentration of Butyl paraben is more than other parabens in water, accounted for $30.45 \%$ of the total in aquatic matter, while concentration of Benzyl paraben is the lowest, accounted for $6.71 \%$. According to the intensity of the suppress of the luminous bacteria, we can characterize the intensity of toxicity of parabens. The results show that Benzyl paraben has the strongest toxic among the five parabens, and has the biggest impact on the environment of lakes in Guangzhou.

Keywords: Lakes; parabens; Guangzhou; GC-MS; toxicity

对差基苯甲酸酯类, 又称为尼泊尔金酯, 常见的包括对差基苯甲酸甲酯 (MPB)、乙酯 (EPB)、丙酯 $(\mathrm{PPB}) 、$ 、酯 $(\mathrm{BPB})$ 和苯甲酯 $(\mathrm{Bz} P \mathrm{~PB})$ 等, 是一类新一代高效低毒的杀菌防腐剂, 因其具有抗菌能力强、 $\mathrm{pH}$ 值 适应范围广、毒性低的特点 ${ }^{[1]}$, 目前被广泛应用于食品、饮料、个人护理用品和药品中 ${ }^{[2-4]}$. 近期一些研究表 明,体香剂中对差基苯甲酸酯类长期的接触可能引发乳腺癌 ${ }^{[5]}$. 对羟基苯甲酸酯类防腐剂已被证明具有雌 激素活性,一旦进人环境可能造成不良影响 ${ }^{[6-8]}$, 并且极易与氯化消毒过的自来水中的余氯发生反应 ${ }^{[9]}$, 主 要生成一氯或二氯、溴代衍生物,这些副产物在大型藻检测中显示出比原始有机物更高的急性毒性 ${ }^{[10]}$, 对环 境和生物造成负面影响.

自从对差基苯甲酸酯类的雌激素活性被发现以来, 国外对城市水体中对羟基苯甲酸酯类防腐剂的浓度 检测以及去除研究做了大量的工作. 如 Lee 等 ${ }^{[11]}$ 通过对加拿大 6 大城市中的 8 所污水处理厂的污水进行分 析, 发现处理前污水中对羟基苯甲酸酯类的平均浓度为 $14.60 \mu \mathrm{g} / \mathrm{L}$, 经污水处理厂处理后去除率为

* 国家自然科学基金项目(21377170)资助. 2014-12-22 收稿;2015-08-17 收修改稿. 刘祖发 (1961 ), 男,副教 授,博士;E-mail:eeslzf@ mail.sysu.edu.cn. 
$95.20 \%$; Jonkers 等 ${ }^{[12]}$ 通过对瑞士的 Glatt 河水中对差基苯甲酸酯类的测定, 发现 Glatt 河中 MPB、EPB、PPB、 $\mathrm{BPB}$ 和 $\mathrm{BzPB}$ 的平均浓度分别为 5.00、0.10、0.60、0.30 和 $0 \mathrm{ng} / \mathrm{L}$; Benijts 等 ${ }^{[13]}$ 发现比利时的河水中对差基 苯甲酸酯类的最高浓度达到 $85.00 \mathrm{ng} / \mathrm{L}$ 等. 然而我国对羟基苯甲酸酯类防腐剂的研究起步较晚, 防腐剂浓 度的研究还停留在食品、药物和化妆品等方面, 对于城市水体中防腐剂的研究目前仍未见过报道.

为了研究国内城市水体中对羟基苯甲酸酯类防腐剂的污染状况,本文选取广州市内的 15 个典型湖泊 在非汛期进行采样, 并运用液液萃取-气质联用法对 MPB、EPB、PPB、BPB 和 BzPB 5 种典型防腐剂进行检测 与毒性分析, 以期为广州市湖泊水体的污染控制和水体生态环境的改善提供参考.

\section{1 样品来源与分析方法}

\section{1 采样点分布}

本文针对广州市的湖泊水体进行研究, 对市区内较大的 15 个湖泊分别进行采样(图 1, L1: 海珠湖, L2: 晓港公园,L3:东山湖公园,L4:荔湾湖公园,L5:流花湖公园, L6 : 越秀公园, L7 : 田心新村,L8: 麓湖, L9: 广州 烈士陵园,L10: 广州动物园,L11: 暨南大学,L12:天河公园, L13:华南师范大学, L14: 华南理工大学, L15:华 南农业大学), 采样点涵盖海珠区、越秀区、天河区和白云区, 能直观地反映广州市区内各湖泊的污染状况. 采样时间为 2012 年 12 月 15 、16 日,采样过程严格遵守标准规范, 保证水样不受污染, 数据结果可靠.

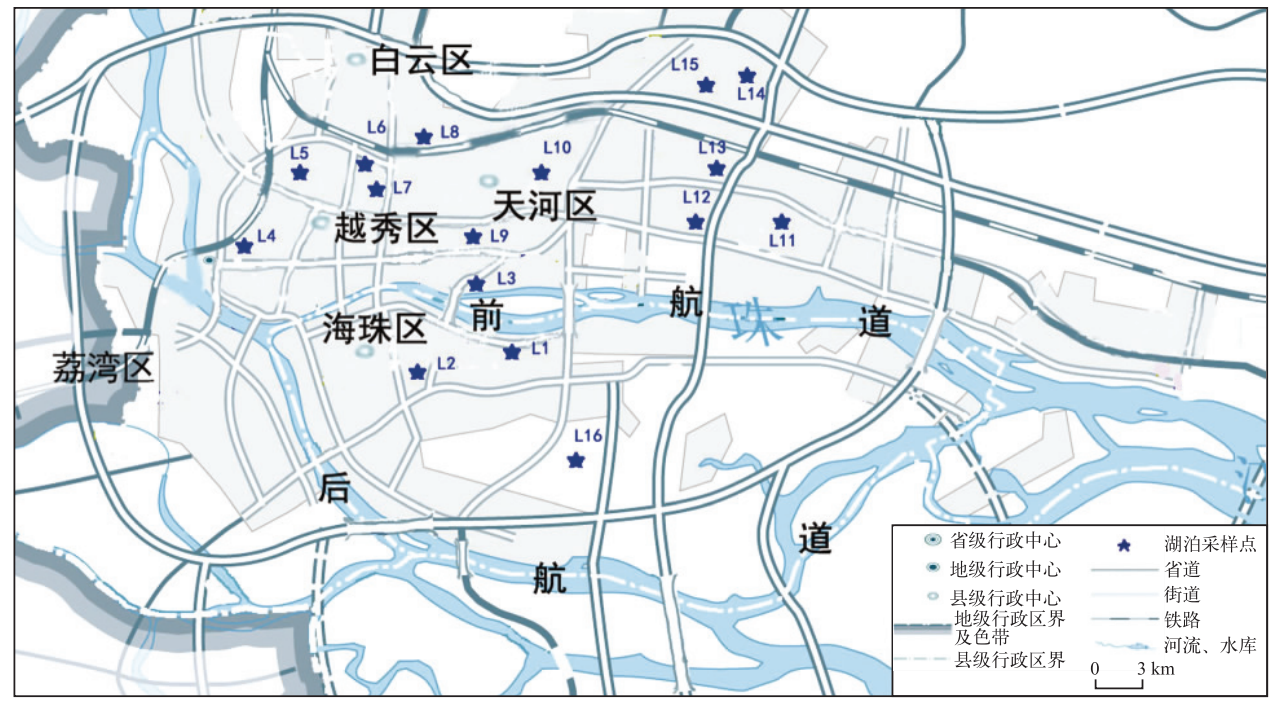

图 1 广州市湖泊采样点分布

Fig.1 Distribution of sampling sites in Guangzhou City

\section{2 样品分析方法}

样品在真空下通过 $0.7 \mu \mathrm{m}$ 孔径的 Whatman GF/F 玻璃纤维膜过滤, 以获得溶解相和颗粒相的样品. 萃 取之前, 每个样品中掺人 $10 \mu \mathrm{l}$ 烷基酚, 用经典的液液萃取法提取样品中的目标物质. 将 $1.0 \mathrm{~L}$ 的水样放置 在分液漏斗中, 以二氯甲烷为萃取剂萃取 3 次, 每次加人 $50 \mathrm{ml}$. 玻璃纤维膜中的颗粒相样品在索氏提取器 中用 $150 \mathrm{ml}$ 二氯甲烷萃取 $24 \mathrm{~h}$. 然后分别对萃取液进行浓缩, 加人 $10 \mathrm{ml}$ 正已烷, 并在温和的氮气流下进一 步浓缩到约 $150 \mu \mathrm{l}$,再向浓缩液中加人 $50 \mu \mathrm{l}$ 的 $\mathrm{N}, \mathrm{O}$-双(三甲基硅基)三氟乙酰胺( BSTFA)与 $1 \%$ 的三甲基 氯硅烷( TMCS) 衍生化, 之后加人 $10 \mu \mathrm{l} 50.00 \mathrm{ng} / \mathrm{ml}$ 的 ${ }^{13} \mathrm{C}-\mathrm{PCB} 208$, 然后用微量进样器准确抽取 $2 \mu \mathrm{l}$ 混合 液, 导人 GC-MS (6890NGC,5973MSD, 美国 Agilent 公司, HP-5MS 毛细管柱) 中测定, 其中进样口衬管型号为 $5183-2037,1.8 \mathrm{~mm}$ ID.

色谱条件: 采用无分流进样, 数据的采集和处理采用 GC-MS solution 软件工作站; 进样口温度为 $280^{\circ} \mathrm{C}$; 柱温程序开始为 $60^{\circ} \mathrm{C}$ 保持 $2 \mathrm{~min}$, 以 $30^{\circ} \mathrm{C} / \mathrm{min}$ 的速率上升至 $130^{\circ} \mathrm{C}$, 之后以 $2^{\circ} \mathrm{C} / \mathrm{min}$ 的速率升温到 $220^{\circ} \mathrm{C}$, 然 
后以 $30^{\circ} \mathrm{C} / \mathrm{min}$ 的速率升温至 $300^{\circ} \mathrm{C}$, 并保持 $5 \mathrm{~min}$.

质谱条件:传输线和离子源温度分别保持在 280 和 $230^{\circ} \mathrm{C}$, 电子能量为 $70 \mathrm{eV}$. 先以保留时间和全扫方式 进行定性分析; 然后根据标液中各物质的出峰时间和特征离子, 采用分时段单扫的方式选择离子, 进行定量 分析.

\section{3 实验参数}

将 5 种标准物质的混合溶液溶解于正已烷中, 分别配置成浓度为 $2 、 5 、 10 、 25 、 50 、 100 \mu \mathrm{g} / \mathrm{L}$ 的溶液, 使用 GC-MS 测定标样. 根据标样的色谱图确定目标化合物的特征离子, 以全扫方式确定出各自的出峰时间 (表1).

表 15 种目标物的特征离子和出峰时间

Tab.1 Characteristic ion and retention time of 5 target compounds

\begin{tabular}{lccccc}
\hline 目标物质 & MPB & EPB & PPB & BPB & BzPB \\
\hline 特征离子 & $209,224,193$ & $223,238,193$ & $210,193,237$ & $210,195,193$ & $193,91,300$ \\
出峰时间 $/ \min$ & 8.316 & 9.816 & 12.406 & 15.680 & 17.260 \\
\hline
\end{tabular}

表 25 种防腐剂的标准曲线及相关系数

Tab.2 Standard curve and correlation coefficient of 5 parabens

\begin{tabular}{ccc}
\hline 目标物 & 标准曲线 & 相关系数 \\
\hline $\mathrm{MPB}$ & $y=0.0357 x$ & 0.9986 \\
$\mathrm{EPB}$ & $y=0.0228 x$ & 0.9991 \\
$\mathrm{PPB}$ & $y=0.0151 x$ & 0.9992 \\
$\mathrm{BPB}$ & $y=0.0238 x$ & 0.9992 \\
$\mathrm{BzPB}$ & $y=0.0316 x$ & 0.9969 \\
\hline
\end{tabular}

根据几种不同浓度的标样进行 GC-MS 分析, 以 目标物与内标物出峰面积的比值为横坐标 $(x)$, 以目 标物与内标物的浓度比值为纵坐标 $(y)$, 得到标准曲 线的方程表达式和相关系数 (表 2). 结果显示, 标准 物质在 2 100 $\mu \mathrm{g} / \mathrm{L}$ 范围内时, 该检测方法得到 5 种 物质的出峰面积比与相应的实际浓度比的相关系数 都在 0.996 以上, 二者具有非常好的线性关系, 可以 据此计算样品中目标物的浓度.

\section{4 实验回收率与检出限}

取 5 种对差基苯甲酸酯的已知浓度溶液, 按照 与样品同样的方法进行处理和 GC-MS 分析, 根据平

行实验得出的结果可以计算标准偏差, 以实验结果的平均值与已知浓度的比值作为该方法的回收率. 根据 美国 EPA 的标准方法, 以 3 倍的信噪比作为计算方法的检出限 $M D L($ method detection limitation ), 即 $M D L=$ $S T D$. 信噪比 ${ }^{[14]}$, 信噪比系数取 3 , 结果表明 5 种目标物质的回收率均在 $90 \%$ 以上, 重复性好, 符合分析要求 (表 3). 因此该方法对 5 种防腐剂的检测限十分理想, 为 $0.01 \sim 0.06 \mathrm{ng} / \mathrm{L}$, 可以在实际水体的检测中进行 应用。

表 3 方法对目标物质的标准偏差、检测限和回收率

Tab.3 Standard deviation, detection limit and recovery of 5 target compounds

\begin{tabular}{lccccc}
\hline 目标物质 & MPB & EPB & PPB & BPB & BzPB \\
\hline 标准偏差 STD & 0.01 & 0.01 & 0.01 & 0 & 0.01 \\
检测限 $/(\mathrm{ng} / \mathrm{L})$ & 0.04 & 0.06 & 0.03 & 0.01 & 0.03 \\
回收率 $/ \%$ & 92 & 90 & 91 & 96 & 98 \\
\hline
\end{tabular}

\section{5 毒性分析方法}

本文采用国内常用的青海湖发光细菌对 5 种防腐剂的毒性进行研究 ${ }^{[15]}$, 以不同物质对青海湖发光细菌 光强的抑制性强弱来表征其毒性的大小. 将对羟基苯甲酸酯的标样以超纯水按梯度进行稀释, 得到一定浓 度梯度的样品. 然后将活化好的青海湖发光细菌放在恒温 $15^{\circ} \mathrm{C}$ 的 LUMIStherm 恒温器中 $15 \mathrm{~min}$ 后待测, 之后 取 $0.5 \mathrm{ml}$ 稀释好的各浓度待测样品加人 $0.5 \mathrm{ml}$ 菌液, 在 $15^{\circ} \mathrm{C}$ 环境的恒温器中接触 $15 \mathrm{~min}$ 后用 $\mathrm{HACH}$ 的 LUMIStox300 毒性检测仪测定出各浓度梯度的样品对发光细菌发光光强的抑制率. 


\section{2 结果与讨论}

\section{1 广州市湖泊水体防腐剂浓度}

使用上述方法对所采集的样品进行萃取和 GC-MS 分析, 检测得到湖泊水体中溶解相和颗粒相中 5 种防 腐剂的总浓度, 结果表明, 广州市内湖泊水体中溶解相的防腐剂浓度范围为 $0.10 \sim 19.40 \mathrm{ng} / \mathrm{L}$, 平均浓度为 $5.06 \mathrm{ng} / \mathrm{L}$ (图 2). 最高值出现在越秀区内的麓湖公园, 这是由于紧挨着麓湖边上分布着几家餐饮店, 餐饮店 产生的污水直接排入麓湖水体, 造成麓湖的防腐剂浓度相对较高; 最低值出现在越秀公园东门附近的湖泊, 这是由于越秀公园是广州省内著名的旅游景点, 平时的管理十分严格, 基本上不会有污水流人的缘故. 相比 之下水体中颗粒相的防腐剂浓度远低于溶解相, 平均浓度为 $0.78 \mathrm{ng} / \mathrm{L}$, 这是由于 5 种防腐剂均微溶于水, 其 在水体中的浓度本身就很低,因此水体颗粒相中的浓度更低.

对羟基苯甲酸酯类除天然存在于一些如蓝莓、白花杜鹃等植物中,其它全部由化学合成以用做防腐剂. 所以未受污染的天然水体中对羟基苯甲酸酯类是不会有检出的,但广州市湖泊水体中对差基苯甲酸酯类防 腐剂的检出率竟高达 100\% (图 2), 这说明广州市内的湖泊水体已经普遍受到防腐剂的污染. 目前国际上还 没有明确的标准来衡量水体中防腐剂的污染水平, 但将本研究结果与 Jonkers 等 ${ }^{[12]}$ 以及 Benijts 等 ${ }^{[13]}$ 的研究 结果作比较,发现广州市内湖泊水体中防腐剂的浓度略低于国外天然水体中的浓度. 其主要原因是广州市 使用对羟基苯甲酸酯类防腐剂的时间相对较短; 其次是由于本次采样的湖泊多数为公园与高校内的湖泊景 观水,有公园和相关部门专门负责, 而且除麓湖外, 其他湖泊几乎不会有生活和工业污水直接汇人.

湖泊水体中防腐剂主要以溶解相存在 (图 2), 所以本文就溶解相中 5 种防腐剂所占比例进行研究, 结果 显示溶解相中 BPB 的平均浓度最高, 占溶解相总量的 $30.45 \%$, 在本文所选取的 15 个湖泊中, 更有 4 个湖泊 水体中 BPB 浓度占 5 种防腐剂溶解相总量的 $58 \%$ 以上; $\mathrm{BzPB}$ 的平均浓度最低, 为 $6.71 \%$, 其中有 7 个湖泊 中 $\mathrm{Bz} P \mathrm{~PB}$ 未检出 (图 3). 另外 MPB 、 $\mathrm{EPB}$ 和 PPB 的平均占比分别为 $25.37 \% 、 7.72 \%$ 和 $29.75 \%$.

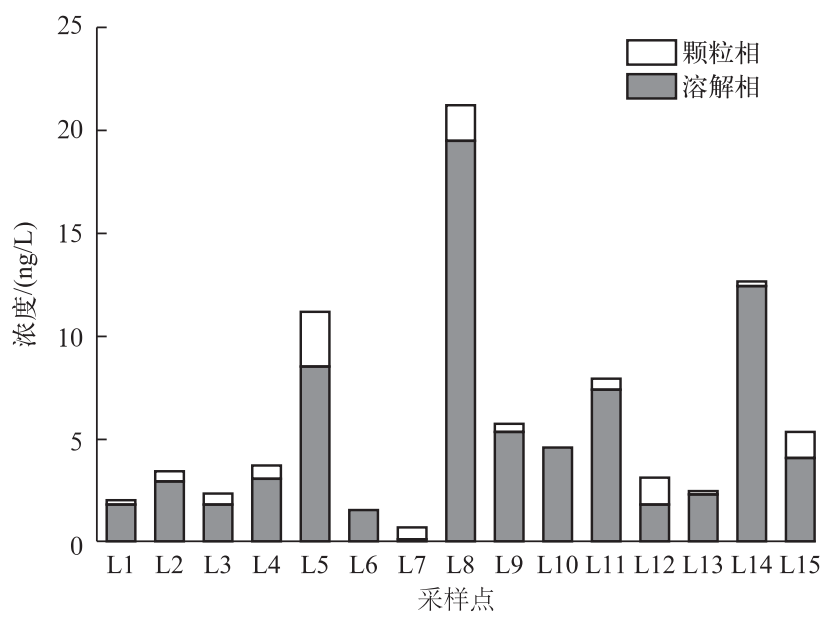

图 2 广州市湖泊水样防腐剂浓度测定结果

Fig.2 Test results of parabens in water samples from lakes in Guangzhou City

\section{2 防腐剂毒性分析}

根据方法 1.5 节得到的结果, 以抑制率为纵轴, 以样品中防腐剂的浓度为横轴, 作出不同浓度的样品与 水样毒性的折线关系图, 实验结果测得水样的毒性随标样浓度的变化如图 4. 由防腐剂毒性分析的折线图可 以看出 (图 4), 5 种防腐剂的毒性均随浓度的升高而上升, 当浓度达到一定限值时其抑制率趋近于 1 , 当浓度 较低时其抑制率趋近于 0 . 通过 LUMIStox300 毒性检测仪的检测, MPB 、EPB、PPB、BPB 和 BzPB 的 $\mathrm{EC}_{50}$ 值分 别为 $5.05 、 6.73 、 12.79 、 8.65$ 和 $0.19 \mathrm{mg} / \mathrm{L}$, 可以看出 5 种对羟基苯甲酸酯类防腐剂中, $\mathrm{BzPB}$ 的毒性最高, 这 与 Applegate 等 ${ }^{[15]}$ 的结论一致. 所以, 虽然自然水体 BzPB 所占的比例最小, 但在研究广州市区防腐剂污染 


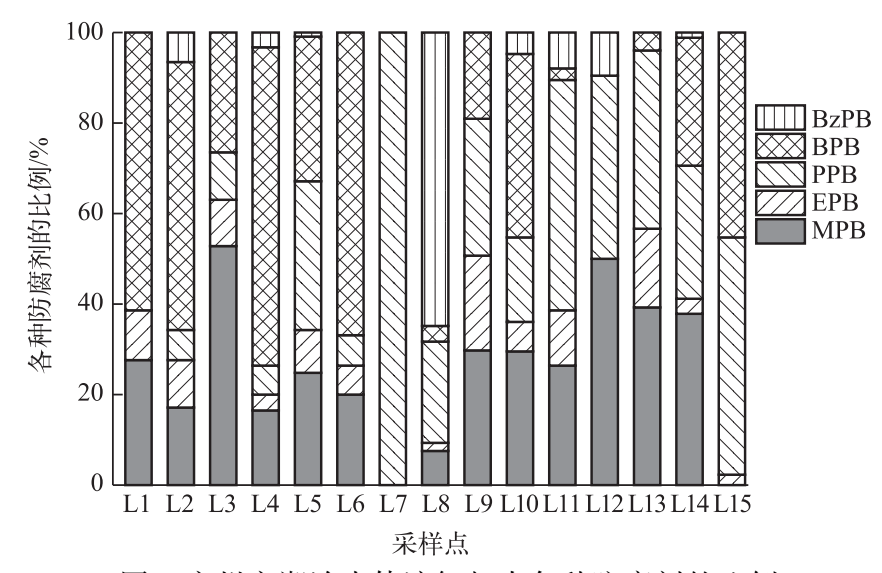

图 3 广州市湖泊水体溶解相中各种防腐剂的比例

Fig.3 Proportions of 5 parabens of dissolved phase in water samples from lakes in Guangzhou City 的问题时, $\mathrm{Bz} P \mathrm{~PB}$ 应得到足够重视.

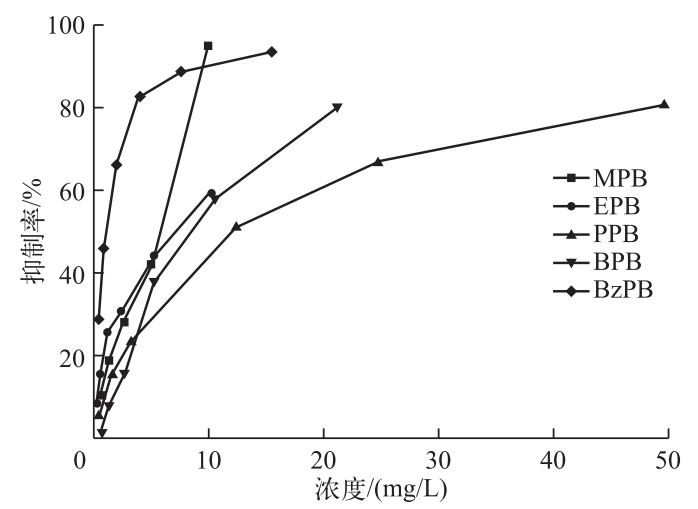

图 4 防腐剂 MPB、EPB、PPB、BPB 和 BzPB 的毒性分析

Fig.4 Analysis of toxicity of MPB,EPB, PPB, BPB and BzPB

\section{3 结论与展望}

1) 对广州市区内 15 个湖泊进行分析检测的结果表明, 水体中防腐剂的检出率高达 $100 \%$, 说明广州市 区内的湖泊普遍受到防腐剂的污染, 其中溶解相的防腐剂平均浓度为 $5.06 \mathrm{ng} / \mathrm{L}$, 颗粒相的平均浓度为 0.78 $\mathrm{ng} / \mathrm{L}$.

2)广州市区的湖泊水体中溶解相防腐剂浓度最高的为对羟基苯甲酸丁酯, 占总量的 30.45\% ; 对差基苯 甲酸苯甲酯所占比例最小, 为 $6.71 \%$.

3) 以防腐剂对发光细菌光强的抑制强弱来表征其毒性的大小, 5 种防腐剂的毒性都随浓度的升高而上 升, 且以对差基苯甲酸苯甲酯的毒性为最强, 考虑到自然水体中所检测到的浓度, 对差基苯甲酸苯甲酯对环 境所产生的影响最大.

本文虽然对广州市内湖泊水体中防腐剂的溶解相和颗粒相的浓度进行研究, 在国内自然水体中防腐剂 的领域上迈出一步, 但对湖泊之外的水体还没有进行研究, 为了准确地了解国内自然水体中防腐剂的分布 与浓度、掌握防腐剂的污染状况, 下一步将对不同类型的自然水体都进行相关研究. 


\section{4 参考文献}

［1］刘志臬,袁亦丞,刘莲芳等. 食品添加剂手册. 北京: 中国轻工业出版社, 1999:201-202.

[ 2 ] Meyer BK, Ni A,Hu B et al. Antimicrobial preservative use in parenteral products: past and present. Journal of Pharmaceutical Sciences, 2007,96(12):3155-3167.

[ 3 ] Lundov MD, Moesby L,Zachariae C et al. Contamination versus preservation of cosmetics: a review on legislation, usage, infections, and contact allergy. Contact Dermatitis,2009,60(2):70-78.

[4] 潘 媛, 李 波, 祝红昆等. 超快速液相色谱测定食品中对羟基苯甲酸酯类. 食品研究与开发, 2011,32(2): 105-107.

[ 5 ] Darbre PD, Aljarrah A, Miller WR et al. Concentrations of Parabens in human breast tumours. Journal of Applied Toxicolo$g y, 2004,24(1): 5-13$.

[ 6 ] Routledge EJ, Parker J, Odum J et al. Some alkyl hydroxy benzoate preservatives (parabens) are estrogenic. Toxicology and Applied Pharmacology, 1998,153(1) : 12-19.

[ 7 ] Golden R, Gandy J, Vollmer G. A review of the endocrine activity of parabens and implications for potential risks to human health. Critical Reviews in Toxicology,2005,35(5):435-458.

[ 8 ] Soni MG, Carabin IG, Burdock GA. Safety assessment of esters of p-hydroxybenzoic acid (parabens). Food and Chemical Toxicology,2005, 43(7) :985-1015.

[ 9 ] Canosa P, Rodri'guez I, Rubi' E et al. Formation of halogenated by-products of parabens in chlorinated water. Analytica Chimica Acta, 2006,575(1):106-113.

[10] Terasaki M, Makino M,Tatarazako N. Acute toxicity ofparabens and their chlorinated by-products with Daphnia magna and Vibrio fischeri bioassays. Journal of Applied Toxicology,2009,29(3) :242-247.

[11] Lee HB, Peart TE, Svoboda ML. Determination of endocrine-disrupting phenols, acidic pharmaceuticals, and personal-care products in sewage by solid-phase extraction and gas chromatography-mass spectrometry. Journal of Chromatography A, $2005, \mathbf{1 0 9 4}(1 / 2): 122-129$.

[12] Jonkers N, Kohler HPE, Dammsha" user A et al. Mass flows of endocrine disruptors in the Glatt River during varying weather conditions. Environmental Pollution, 2009,157(3) :714-723.

[13] Benijts T, Lambert W, De Leenheer A. Analysis of multiple endocrine disruptors in environmental waters via wide-spectrum solid-phase extraction and dual-polarity ionization LC-ion trap-MS/MS. Analytical Chemistry, 2004,76(3):704-711.

[14] 刘祖发,张素琼,卓文珊等. 广州市海珠区地表水中致嗅物质及毒性分析. 湖泊科学, 2013,25(6): 900-906. DOI 10.18307/2013.0614.

[15] Applegate VC, Howell JH, Hall AE et al. Toxicity of 4,346 chemicals to larvallampreys and fishes. Special Scientific Report, Fisheries No. 207,U. S. Fish and Wildlife Service, Department of the Interior, Washington DC: 1957:157. 